\title{
Bistability in Semiconductor Lasers With Polarization-Rotated Frequency-Dependent Optical Feedback
}

\author{
C. Masoller, T. Sorrentino, M. Chevrollier, and M. Oria
}

\begin{abstract}
Bistability in the emission frequency of a semiconductor laser subject to orthogonal-polarization optical feedback was recently observed experimentally by Farias et al. in 2005. A frequency-sensitive filter (Cs-vapor cell) was placed in the way of the feedback beam to spectrally modulate the feedback power. Two different emission frequencies with the same output power were observed. This observation was understood in terms of a model that took into account the line shape of the filter and the empirical linear relation between the feedback-induced frequency shift and the feedback intensity. The model allowed to calculate steady states but not time-varying solutions. Here we present a rate-equation model that takes into account thermal and gain-saturation effects, and predicts a linear variation of the laser frequency with the feedback strength, together with a small power modulation. This model allows to study time-dependent solutions, and in particular, the transition between the two coexisting states. We show that numerical simulations using this model correctly reproduce the previous observed dynamics, and we present new experimental results in good agreement with our model for the laser response under orthogonal feedback.
\end{abstract}

Index Terms-Bistability, optical feedback, semiconductor lasers.

\section{INTRODUCTION}

$\mathbf{S}$ EMICONDUCTOR lasers are key elements of today's optical communications technology and have immense importance in consumer electronics, being used in CD and DVD players, printers, scanners, etc. Under external perturbations, such as optical feedback, optical injection and modulation, these lasers often present instabilities in their optical output. The influence of optical feedback has received specific attention, because it can lead either to an improved performance (mode-hopping suppression and linewidth reduction), or to unstable emission (in the form of low-frequency fluctuations and coherence collapse) [1]-[3].

Recent work has focused on the influence of orthogonal feedback, because it can lead to novel applications [4]-[7]. In this

Manuscript received August, 2006; revised November, 2006. This work was supported in part by the Brazilian agencies FINEP under the Fenix Project, CAPES-COFECUB under Project 456/04, and CNPq. The work of C. Masoller was supported in part by the "Ramon and Cajal" Program (Spain).

C. Masoller is with the Departament de Fisica i Enginyeria Nuclear, Universitat Politecnica de Catalunya, E-08222 Terrassa, Spain (e-mail: cristina. masoller@upc.edu).

T. Sorrentino, M. Chevrollier, and M. Oria are with the Laboratório de Física Atômica e Lasers, Universidade Federal da Paraíba, 58051-970 João Pessoa-PB, Brazil (e-mail: oria@otica.ufpb.br).

Digital Object Identifier 10.1109/JQE.2006.889647 scheme the polarization state of the feedback light is rotated 90 degrees such that the natural laser mode (which we refer to as TE), is coupled to the orthogonal unsupported mode (which we refer to as TM). Antiphased square-wave oscillations of the intensities of the two orthogonal polarizations were recently observed by Gavrielides and co-workers [8]. Polarization selfmodulation can have applications for the generation of optical pulses at high-repetition rates (not limited by the relaxation oscillation frequency) without the need for high-speed electronics, as already reported by Loh et al. in 1990 [9], [10]. Based on a rate-equation model that considers the amplitudes of both orthogonal modes, Gavrielides et al. [8] found that these oscillations appear for a broad range of parameters, provided that the feedback is strong and the differential losses in the unsupported TM mode are small. In vertical-cavity surface-emitting lasers (VCSELs) the dominant polarization mode and the orthogonal mode have similar losses (because of the cylindrical transverse geometry), and thus VCSELs are good candidates for generation of high-speed polarization self-modulation [11]. The synchronization of two lasers with orthogonal feedback and/or orthogonal coupling has also been studied by several groups [12]-[16], and it was reported that it has the advantage, with respect to the case of conventional optical feedback, that it does not require a careful matching of the emission frequencies of the two lasers.

Recently, Oria and co-workers [17], [18] studied the influence of frequency-selective orthogonal feedback, placing a frequency-sensitive filter (Cs-vapor cell) in the way of the feedback beam to spectrally modulate the feedback power. Two different emission frequencies with almost the same output power were observed (less than 1\% amplitude modulation was observed). This frequency bistability can have potential applications for all-optical logic gates and optical digital information technologies. The bistability found was understood in terms of a model that took into account the line shape of the filter and the empirical linear relation between the feedback-induced frequency shift and the feedback intensity. The model allowed to calculate steady-states but not time-varying solutions.

In this paper we present a rate-equation model that, taking into account thermal and gain-saturation effects, predicts a linear variation of the laser frequency with the feedback strength. The model allows to study time-dependent solutions, and in particular, the transition between the two coexisting states. We show that numerical simulations using this model correctly reproduce the previous observed dynamics, and we present additional experimental observations that are in good agreement with the predictions of the model. 
This paper is organized as follows. Section II presents the model equations. It is shown that, by including thermal and gain saturation effects in the conventional laser rate equations, the model predicts a linear frequency shift of the emission frequency with the orthogonal feedback strength. Results of numerical simulations are presented in Section III. It is shown that when the feedback strength is frequency-dependent, there is bistability for large enough feedback, and the size of the bistable region increases with the feedback strength, in good agreement with observations of [18]. Moreover, it is shown that thermal effects lead to an intrinsic hysteresis cycle, which is observed even in the absence of orthogonal feedback. Section IV presents additional experimental measurements that confirm the predictions of the model. Section V presents a summary and the conclusions.

\section{MODEL}

The slowly varying complex amplitude of the laser intracavity field with TE polarization, $\mathcal{E}$, the carrier density $\mathcal{N}$, and the lattice temperature $\mathcal{T}$ are described by a set of rate equations [19], [20], extended to account for orthogonal optical feedback

$$
\begin{aligned}
\frac{d \mathcal{E}}{d t} & =i k \theta \mathcal{E}+k(1+i \alpha)(\Gamma G-1) \mathcal{E} \\
\frac{d \mathcal{N}}{d t} & =-\gamma_{N}\left[\mathcal{N}-J+G\left(\mathcal{P}+\mathcal{P}_{f}\right)\right] \\
\frac{d \mathcal{T}}{d t} & =-\gamma_{T}\left(\mathcal{T}-1-Z \mathcal{N}-P J^{2}\right)
\end{aligned}
$$

Here the variables are normalized such that $\mathcal{E}$ is dimensionless, $\mathcal{N}$ is normalized to the transparency value and $\mathcal{T}$ is normalized to the ambient temperature. $\mathcal{E}, \mathcal{N}$, and $\mathcal{T}$ decay rates are $k, \gamma_{N}$ and $\gamma_{T}$, respectively.

In the field equation, $\theta$ is a detuning that varies linearly with the temperature

$$
\theta=\theta_{0}-\epsilon(\mathcal{T}-1)
$$

where $\epsilon$ is the coupling strength between the optical field and the temperature. Equation (4) incorporates the main effect of heating in semiconductor devices: a change in the background refractive index and hence a shift in the cavity resonances. Other parameters in (1) are the linewidth enhancement factor, $\alpha$, the optical confinement factor to the active region $\Gamma$ and the gain, which for the moment we assume to be linear $G(\mathcal{N})=\mathcal{N}-$ 1. We will show below that, in order to explain the feedbackinduced frequency shift observed in [18], it will be necessary to include gain saturation terms.

In the carrier density equation, $J=I /\left(\gamma_{N} e N_{0} V\right)$ is the current density, with $I$ being the bias current, $V$ the volume of the active region, $N_{0}$ the transparency density and $e$ the electron charge. The term $\mathcal{P}$ represents the density of photons with TE polarization, $\mathcal{P}(t)=|\mathcal{E}(t)|^{2}$, and the term $\mathcal{P}_{f}$ represents the density of photons with TM polarization

$$
\mathcal{P}_{f}(t)=\kappa|\mathcal{E}(t-\tau)|^{2}
$$

where $\kappa$ is the feedback strength and $\tau$ is the delay time, $\tau=$ $2 L / c$, with $L$ being the length of the external cavity and $c$ the velocity of light. When a frequency-sensitive filter is placed in the way of the feedback beam, the feedback strength depends on the frequency $\omega_{\tau}$ of the delayed field

$$
\kappa=\kappa_{0}\left[1-f\left(\omega_{\tau}\right)\right]
$$

where $\kappa_{0}$ stands for the attenuation in the different optical elements of the feedback loop when $\omega_{\tau}$ is nonresonant with the filter resonance frequency $\omega_{\text {at }}$ which is equal to the reference frequency $\omega_{0}$ used for making the slowly varying amplitude approximation. The delayed frequency is calculated self-consistently from the condition $\omega_{\tau}=\operatorname{Im}[\cdot E(t-\tau) / E(t-\tau)]$.

In the temperature equation, the terms $Z \mathcal{N}$ and $P J^{2}$ describe the heating of the device due to nonradiative recombination and due to the Joule effect, respectively.

We begin by searching for steady-state solutions of the form $\mathcal{E}(t)=E e^{i \omega t}, \mathcal{N}(t)=N, \mathcal{T}(t)=T$. Substituting in (1)-(3) and using $\mathcal{P}_{f}=\kappa|E|^{2}$ gives

$$
\begin{aligned}
\Gamma G & =1 \\
N & =1+1 / \Gamma \\
|E|^{2} & =\frac{\Gamma(J-N)}{1+\kappa} \\
T & =1+Z N+P J^{2}, \\
\omega & =k \theta=k \theta_{0}-k \epsilon\left(Z N+P J^{2}\right) .
\end{aligned}
$$

We see that the TE photon density decreases with increasing feedback strength while the carrier density, the lasing threshold, $J_{\text {th }}=1+1 / \Gamma$, the temperature, and the emission frequency are not modified by the feedback.

However, in the experiments [18] it is observed that orthogonal feedback modifies the emission frequency of the laser, and moreover, there is a linear dependence of the frequency shift with the fraction of the power sent back to the laser $\mathcal{P}_{f}$

$$
\omega-\omega_{\mathrm{sol}}=-2 \pi \beta \mathcal{P}_{f}
$$

where $\omega$ is the emission frequency of the laser under orthogonal feedback, $\omega_{\text {sol }}$ is the emission frequency of the solitary laser, and $\beta$ is a coefficient that in the experiments [18] was found to depend on the precise alignment of the feedback $(\beta=1.76$ $\mathrm{GHz} / \mathrm{mW}$ when $\mathcal{P}_{f}$ is measured in milliwatts).

Next, let us show that when gain saturation effects are taken into account, the model predicts the linear relation of the frequency shift versus the feedback strength, (12). We consider an optical gain of the form

$$
G\left(\mathcal{N}, \mathcal{P}, \mathcal{P}_{f}\right)=(\mathcal{N}-1) /\left(1+\epsilon_{1} \mathcal{P}+\epsilon_{2} \mathcal{P}_{f}\right)
$$

where $\epsilon_{1}$ and $\epsilon_{2}$ are self- and cross-saturation coefficients, respectively. Self-saturation takes into account various effects such as spectral and spatial hole burning, cross-saturation is mainly due to spatial effects and it is determined by the spatial overlap of the feedback transverse optical profile and the carrier profile in the active region of the laser.

The steady-state solutions verify $\Gamma G=1$, and thus, the steady-state carrier density is

$$
N=1+\left[1+\left(\epsilon_{1}+\epsilon_{2} \kappa\right)|E|^{2}\right] / \Gamma \text {. }
$$


TABLE I

LASER AND FILTER PARAMETERS

\begin{tabular}{lcr} 
Value & Parameter & Description \\
\hline $300 \mathrm{~ns}^{-1}$ & $k$ & Cavity decay rate \\
$1 \mathrm{~ns}^{-1}$ & $\gamma_{N}$ & Carrier decay rate \\
$0.005 \mathrm{~ns}^{-1}$ & $\gamma_{T}$ & Temperature decay rate \\
$6 \mathrm{~ns}$ & $\tau$ & Delay time \\
3 & $\alpha$ & Linewidth enhancement factor \\
0.9 & $\Gamma$ & Optical confinement factor \\
0.3 & $Z$ & Nonradiative recombination heating \\
0.0005 & $P$ & Joule heating \\
200 & $\epsilon$ & Detuning thermal rate \\
0.01 & $\epsilon_{1}$ & Self-saturation coefficient \\
0.019 & $\epsilon_{2}$ & Cross-saturation coefficient \\
0.5 & $\epsilon_{0}$ & Absorption coefficient at resonance \\
$7.7 \mathrm{GHz}^{-2}$ & $\alpha_{0}$ & Gaussian width coefficient
\end{tabular}

From (11), the feedback-induced frequency shift is

$$
\omega-\omega_{\mathrm{sol}}=-k \epsilon Z\left(N-N_{\mathrm{sol}}\right)
$$

where $N_{\text {sol }}$ is the carrier density without feedback. To calculate $N$ and $N_{\text {sol }}$ we need to solve (9) and (14), which give

$$
\begin{aligned}
|E|_{\mathrm{sol}}^{2} & =\frac{\Gamma\left(J-J_{\mathrm{th}}\right)}{1+\epsilon_{1}} \\
N_{\mathrm{sol}} & =J-|E|_{\mathrm{sol}}^{2} / \Gamma \\
|E|^{2} & =\frac{\Gamma\left(J-J_{\mathrm{th}}\right)}{1+\epsilon_{1}+\kappa\left(1+\epsilon_{2}\right)} \\
N & =J-(1+\kappa)|E|^{2} / \Gamma .
\end{aligned}
$$

Substituting in (15), the feedback-induced frequency shift is

$$
\begin{aligned}
\omega-\omega_{\mathrm{sol}} & =-k \epsilon Z\left[-(1+\kappa)|E|^{2}+|E|_{\mathrm{sol}}^{2}\right] / \Gamma \\
& =-\frac{k \epsilon Z \kappa\left(\epsilon_{2}-\epsilon_{1}\right)}{\Gamma\left(1+\epsilon_{1}\right)}|E|^{2} \\
& =-\frac{k \epsilon Z\left(\epsilon_{2}-\epsilon_{1}\right)}{\Gamma\left(1+\epsilon_{1}\right)} \mathcal{P}_{f}
\end{aligned}
$$

and it can be noticed that it varies linearly with the fraction of the power sent back to the laser, $\mathcal{P}_{f}$, in agreement with the experimental observations [18]. We notice that the coefficient $\beta=k \epsilon Z\left(\epsilon_{2}-\epsilon_{1}\right) / \Gamma\left(1+\epsilon_{1}\right)$ is independent of the bias current, also in good agreement with the observations. We also note that $\beta>0$ if $\epsilon_{2}>\epsilon_{1}$.

\section{NUMERICAL RESULTS}

In this section, we show that for a reasonable choice of parameter values our model gives results that are in good agreement with the observations of [18]. Unless otherwise stated the parameters are those summarized in Table I: the decay rates $k, \gamma_{N}, \gamma_{T}$, and the $\alpha$ factor are typical values for semiconductor lasers; the other parameters are chosen as described below, to fit the experimental situation in [18].

To use the experimental value $\beta=1.76 \mathrm{GHz} / \mathrm{mW}$ in (20), first we have to convert the dimensionless photon density, $\mathcal{P}_{f}$, to power measured in milliwatts. This can be done using the linear relation between the output power and the bias current less the threshold current, $I-I_{\text {th }}$. In [18] the output power for a bias current of $I \sim 2.3 I_{\mathrm{th}}$ is $\sim 40 \mathrm{~mW}$. Thus

$$
\begin{aligned}
\mathcal{P}(\mathrm{mW})= & \frac{40(\mathrm{~mW})}{1.3 I_{\mathrm{th}}}\left(I-I_{\mathrm{th}}\right) \\
& =\frac{40(\mathrm{~mW})}{1.3 J_{\mathrm{th}}}\left(J-J_{\mathrm{th}}\right) \\
= & \frac{40(\mathrm{~mW})\left(1+\epsilon_{1}\right)}{1.3 J_{\mathrm{th}} \Gamma} \mathcal{P}(\operatorname{adim})
\end{aligned}
$$

where we have used (16). Substituting in (12) and comparing with (20) we have

$$
k \in Z\left(\epsilon_{2}-\epsilon_{1}\right)=2 \pi \beta 40(\mathrm{~mW})\left(1+\epsilon_{1}\right)^{2} /\left(1.3 J_{\mathrm{th}}\right) .
$$

To find adequate parameters we also have to take into account that in [18] the solitary emission frequency varies linearly with the bias current

$$
\omega_{\mathrm{sol}}-\omega_{\mathrm{th}}=-2 \pi K\left(I-I_{\mathrm{th}}\right)
$$

with $K=3.75 \mathrm{GHz} / \mathrm{mA}$. To use this value of $K$ we need to convert the bias current $I$ measured in $\mathrm{mA}$ to dimensionless current density $J=I /\left(\gamma_{N} e N_{0} V\right) \cdot N_{0} V$ can be estimated by making the threshold condition predicted by the model, $J_{\mathrm{th}}=$ $1+1 / \Gamma$, agree with the experimental value, $I_{\mathrm{th}}=43.5 \mathrm{~mA}$. With $\Gamma=0.9, \gamma_{N}=1 \mathrm{~ns}^{-1}$ and $e=1.6 \times 10^{-19} \mathrm{C}$ we obtain $N_{0} V=1.29 \times 10^{8}$.

From (11) we have

$$
\begin{aligned}
\omega_{\mathrm{sol}}-\omega_{\mathrm{th}} & =-k \epsilon\left[Z\left(N_{\mathrm{sol}}-N_{\mathrm{th}}\right)+P\left(J^{2}-J_{\mathrm{th}}^{2}\right)\right] \\
& =-k \epsilon\left[\frac{Z \epsilon_{1}}{1+\epsilon_{1}}\left(J-J_{\mathrm{th}}\right)+P\left(J^{2}-J_{\mathrm{th}}^{2}\right)\right]
\end{aligned}
$$

where we have used $N_{\mathrm{th}}=J_{\mathrm{th}}$ and (16) and (17). Due to Joule heating, the curve $\omega_{\text {sol }}$ versus $J$ is linear only over a limited current range. The nonlinearity is however small, as typically $P \ll Z$ [19], [20]. The parameters $\left(\epsilon, Z, P, \epsilon_{1}, \epsilon_{2}\right)$ given in Table I correspond to $\beta=1.76 \mathrm{GHz} / \mathrm{mW}$ and $K=3.75$ $\mathrm{GHz} / \mathrm{mA}$, and are used in all the simulations, unless otherwise stated.

As in the experiments, in the simulations the feedback strength $\kappa$ depends of the frequency of the feedback field, $\kappa=\kappa_{0}\left[1-f\left(\omega_{\tau}\right)\right]$, and the spectral shape of the filter is assumed to be Gaussian

$$
f(\omega)=\epsilon_{0} \exp \left[-\alpha_{0}\left(\omega-\omega_{\mathrm{at}}\right)^{2}\right],
$$

where $\alpha_{0}$ measures the width of the Gaussian and $\epsilon_{0}$ is the absorption coefficient at resonance. The filter resonance frequency, $\omega_{\text {at }}$, is equal to the solitary laser frequency when the injection current is $100 \mathrm{~mA}$. To scan the laser frequency up-down near the resonance, the bias current was modulated by a triangular signal, varying from 97 to $101 \mathrm{~mA}$. The total time interval of the scan is $\Delta t=72 \mu \mathrm{s}$, unless otherwise stated. While in the experiments a slower current ramp was employed (as discussed in the next section), numerically we observe similar results using a slower ramp and a slower variation of the temperature. In other words, the numerical results do not change when 

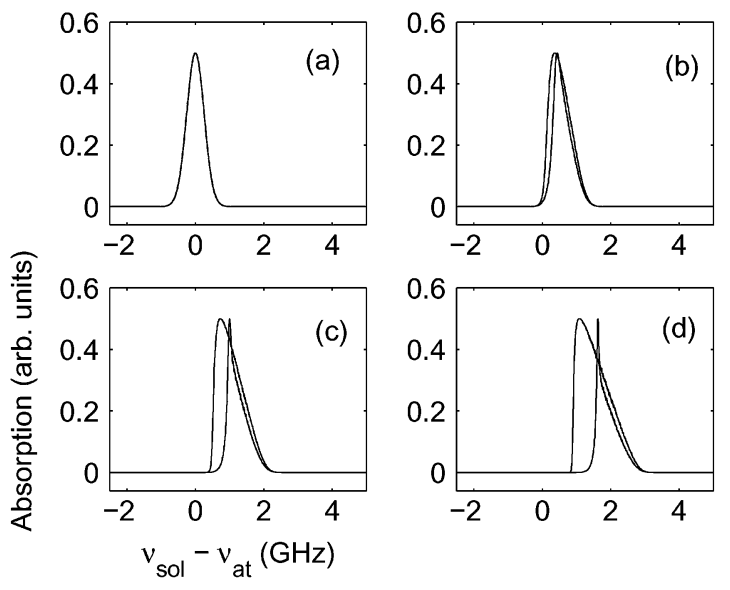

Fig. 1. Filter response versus the solitary laser emission frequency. The feedback strength $\kappa_{0}$ is (a) 0 , (b) 0.01 , (c) 0.02 , and (d) 0.03 .
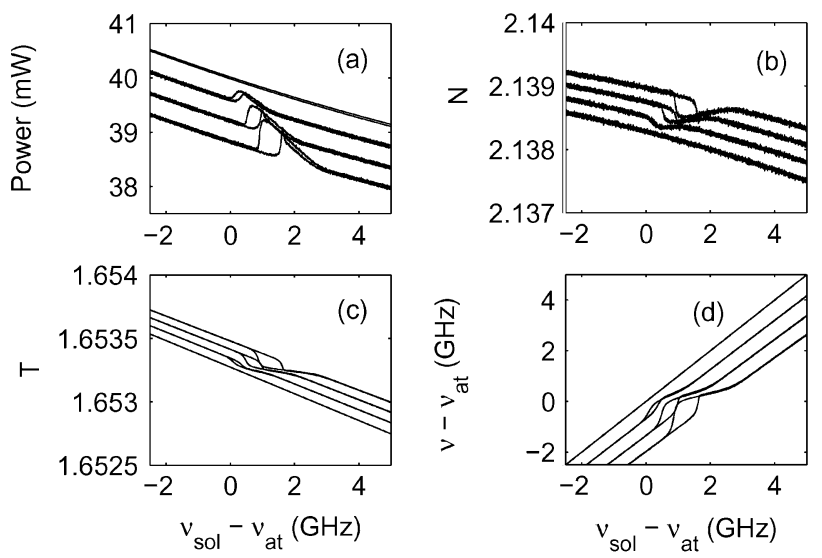

Fig. 2. (a) Laser output power versus the solitary laser emission frequency. The feedback strength is (from top to bottom) $0,0.01,0.02$, and 0.03. (b) Carrier density normalized to the transparency value $N_{0}$. (c) Temperature normalized to the ambient temperature $T_{0}$. (d) Emission frequency.

using larger $\Delta t$ and smaller $\gamma_{T}$. To avoid long time-consuming simulations, we choose the value of $\gamma_{T}$ presented in Table I. A further fine tuning of parameters for achieving better agreement with the experiments does not appear to be useful, since there are several unknown parameters. Therefore, we prefer to demonstrate the robustness of the observed features assuming reasonable parameters.

Fig. 1(a) displays the filter absorption $f(\omega)$ in the absence of feedback, and Fig. 1(b)-(d) displays $f(\omega)$ for three increasing feedback levels. A clear bistability domain can be seen, whose size increases with the feedback strength. Fig. 2 displays the corresponding evolution of the output power Fig. 2(a), carrier density Fig. 2(b), temperature Fig. 2(c) and emission frequency Fig. 2(d). When the laser frequency is scanned up-down the filter resonance there is a frequency jump of $\sim 1 \mathrm{GHz}$ (for $\kappa_{0}=0.03$ ), accompanied by a small variation of $\mathcal{P}$ and there is almost no observable variation of $\mathcal{N}$ and $\mathcal{T}$. These results are in very good agreement with the observations of [18].

In Fig. 2(c), it can be noticed that the value of the operating temperature, about 1.6 times the ambient temperature, is unrealistically high. However, simulations show that the temperature can be decreased to more realistic values by the simultaneous
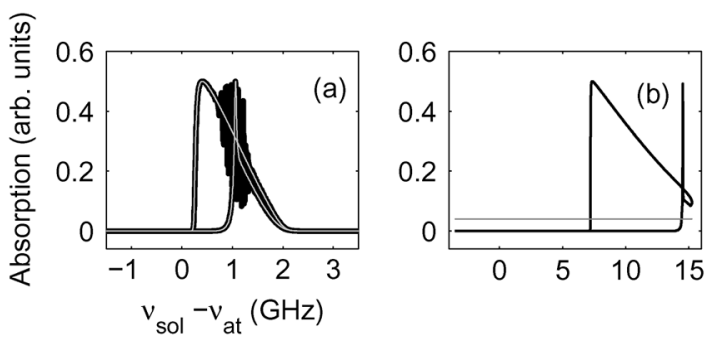

Fig. 3. Absorption for strong feedback. (a) $\kappa_{0}=0.04$, the response of the filter depends on the instantaneous frequency (black line), and the response of the filter depends on the average frequency (averaged over an interval of $0.1 \mathrm{~ns}$, gray line). (b) $\kappa_{0}=0.2$ (black line) and $\kappa_{0}=0.25$ (gray line, shifted vertically for clarity).
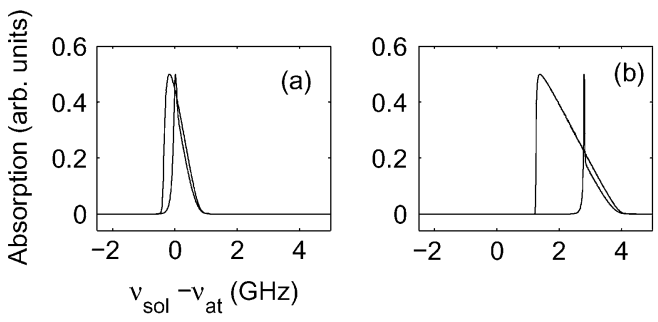

Fig. 4. Absorption for (a) $\beta=0.54 \mathrm{GHz} / \mathrm{mA}$ and $\mathcal{P}_{f}=2.41 \mathrm{~mW}\left(\kappa_{0}=\right.$ $0.063) . \epsilon_{2}=0.013$, other parameters are as in Table I. (b) $\beta=1.23 \mathrm{GHz} / \mathrm{mA}$ and $\mathcal{P}_{f}=4 \mathrm{~mW}\left(\kappa_{0}=0.11\right) . \epsilon_{2}=0.016$, other parameters are as in Table I.

variation of other parameters, such as $\epsilon$ in (4), the field decay rate $k$ in (1) and the thermal decay rate $\gamma_{T}$ in (3). Notice that the relevant temperature variations are very small, therefore, the laser operates at an almost constant temperature, in good agreement with the experiments.

For stronger feedback, instabilities arise and there are oscillations of the laser variables, not only when the injection current varies in time, but also when $J$ is constant. The instability originates on the frequency dependence of the feedback strength: a stable output is observed for these values of $J$ and $\kappa$ if $\kappa$ is independent of $\omega$. Notice that in (25) the feedback strength depends on the instantaneous frequency of the feedback field, which can present fast fluctuations for strong enough feedback, but if we include phenomenologically a "slow response" of the atomic filter, by allowing the absorption to depend on the time averaged optical frequency, then the instabilities disappear and a stable output is observed even for large feedback strengths, as shown in Fig. 3. For stronger feedback the resonance moves to the right and if the current scan is not long enough the switching point is not reached and the resonance is not seen; the atomic filter becomes transparent, as shown by the gray line in Fig. 3(b) (shifted vertically for clarity).

Let us now investigate the influence of the parameter $\beta$ that measures the feedback-induced frequency shift. Fig. 4 displays results for various $\beta$, that reproduce the different experimental conditions presented in the following, experimental section, demonstrating the robustness of the above observed feedback-induced features.

Finally, let us mention that the simulations clearly show the existence of a hysteresis cycle even in the absence of orthogonal feedback. The hysteresis is due to thermal effects: the slow variation of the temperature with the injection current when the 


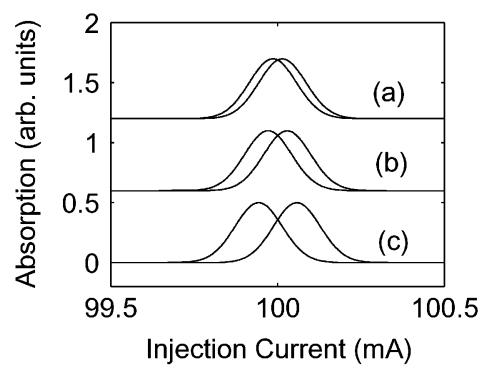

Fig. 5. Hysteresis in the absence of orthogonal feedback, for different velocities of the current ramp. The injection current is varied, to scan the laser frequency up-down across the filter resonance frequency, in a time interval $\Delta T=$ (a) 144 , (b) 72 , and (c) $36 \mu \mathrm{s}$.

current is scanned up-down, prevents the emission frequency to reach its equilibrium steady-state value; therefore, the value of $\omega$ for a given $J$ when $J$ is increasing differs from the value of $\omega$ for the same $J$ when $J$ is decreasing, giving rise to shifted absorption curves for increasing and decreasing $J$, as shown in Fig. 5. As expected, the hysteresis cycle depends on the velocity of current ramp, the faster the current ramp, the larger the hysteresis cycle, and vice-versa, the slower the current ramp, the closest the frequency is to its equilibrium value, and thus, there is only a small hysteresis. The hysteresis cycle is expected only in a restricted range of current ramp frequencies, such that the ramp is fast enough to prevent the emission frequency to reach its equilibrium value, but is slow enough to allow for diffusion processes and temperature variations. We remark however that, in experiments, controlling the laser temperature does not necessarily suppress the hysteresis, because, as mentioned above, the temperature variations involved in this effect are very small and might take place even in temperature-controlled devices (depending on the precision of the cooling system).

\section{EXPERIMENTAL MEASUREMENTS}

The experimental setup, displayed in Fig. 6, is essentially the same as described in [18] and we remind here its main aspects: part of the output power of a laser (Fabry-Pérot type, AsAlGa junction) is sent back into the semiconductor cavity with its polarization rotated $90 \mathrm{deg}$. A polarizer (Glan-Foucault, rejection of $50 \mathrm{~dB}$ ) guarantees that the feedback beam is orthogonal to the outgoing beam. The power sent back into the laser is modulated by a resonant filter, the Doppler broadened $D_{2}$ line of a warm cesium atomic vapor. When the laser frequency is scanned around the atomic transition we observe a frequency behavior as theoretically described above.

Let us emphasize other characteristics of the experimental setup.

- The atomic resonance is observed with a laser operation current around $100 \mathrm{~mA}$, corresponding to an output power of $40 \mathrm{~mW}$, essentially in the main polarization of the laser (TE mode). The intensity in the orthogonal direction (TM mode) is 800 times lower.

- The feedback loop is almost $2 \mathrm{~m}$ long giving a delay time of $6 \mathrm{~ns}$.

- The vapor density of the $20-\mathrm{mm}$-long cell is controlled by the temperature of the cell reservoir, and densities are

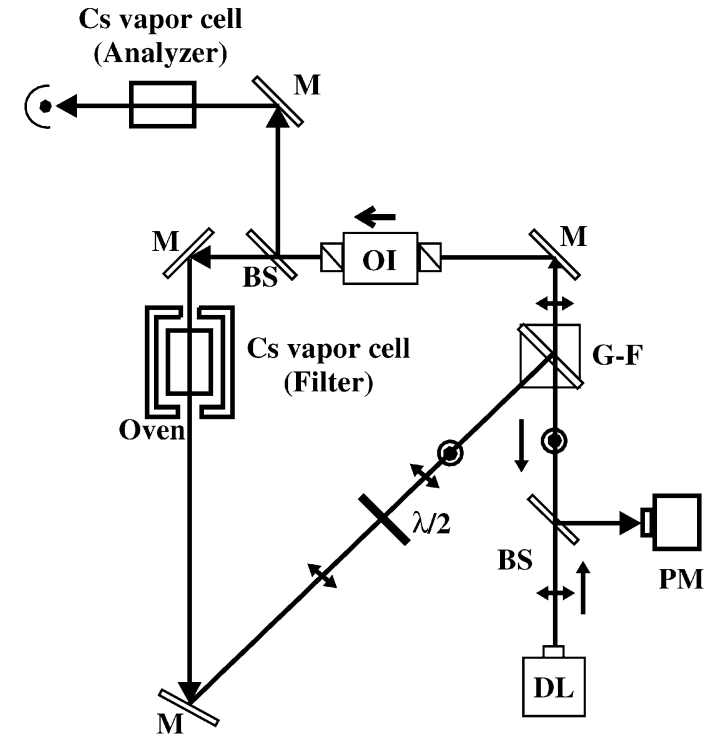

Fig. 6. Experimental setup. The laser beam is sent through a polarizer (G-F): the TM-polarization field $(\odot)$ is transmitted, filtered, and sent back into the laser with a polarization rotated by a half-wave plate $(\lambda / 2)$. The G-F polarizer only reflects the TE component $(\longleftrightarrow)$. A power meter $(\mathrm{PM})$ measures the power of the feedback beam. Other elements—BS: beam splitter; M: mirror; OI: optical isolator.

varied around $10^{13}$ atoms $/ \mathrm{cm}^{3}$. It allows a good filtering for relatively high powers of the feedback beam (up to $5 \mathrm{~mW}$ ).

- Around the operation current the laser output power is stable (within $10^{-3}$ ) even in the presence of feedback, which opens the way to explore exclusive dynamical behavior in the frequency domain.

- A second Cs-vapor cell is used, in order to probe the frequency of the laser emission. This frequency Analyzer (see Fig. 6) is out of the optical feedback loop.

- The laser temperature (at around $18^{\circ} \mathrm{C}$ ) is controlled within a precision of $10^{-4}$.

In Figs. 7 and 8, we present spectra of the analyzer transmission for different levels of the orthogonal feedback power. In both series the spectra present lineshapes and hysteresis ranges that are in good agreement with the calculated ones (see Fig. 4). Notice that the coupling of the feedback beam and the laser gain volume is different in the two series, which are well reproduced by the calculated spectra (see Figs. 1 and 4). We recall that the coefficient is experimentally obtained by measuring the frequency shift as a function of the feedback power. The experimental control of this coupling was done through a very sensitive, but precise and reproducible manipulation in the alignment of the beam returned into the semiconductor junction. This control has also an important role in the multistability lineshapes, as discussed in [25].

Using stronger feedback beam, besides the bistability range increasing we observe sharper frequency transitions from the unstable to the stable flanks, as has been qualitatively explained in [17]. Notice that in the absorption spectra shown in Fig. 8 the amplitude of the narrow peak appears to be smaller than the larger one. However, this is an effect of the scanning averaging for this very narrow peak. Slowing the scanning we get higher amplitudes for this peak, as shown in Fig. 9, but at the cost of having noisier spectra. In this figure we show two different conditions (different $\beta$ and feedback power). In the particular case 


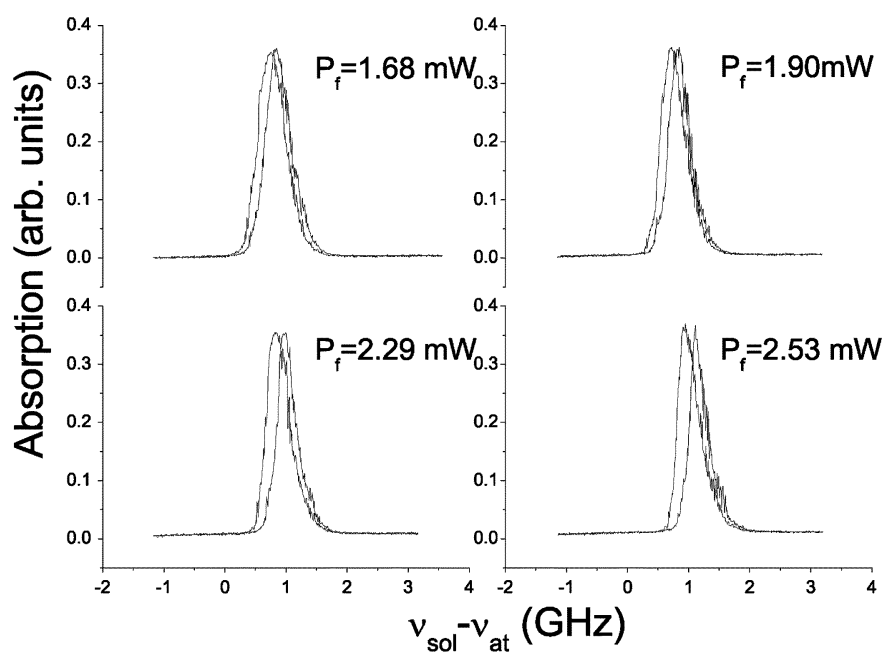

Fig. 7. Absorption spectra for low feedback power $\mathcal{P}_{f} \cdot \mathcal{P}_{f}$ values are indicated at the top of each spectrum. $\beta$ is $0.54 \mathrm{GHz} / \mathrm{mW}$.

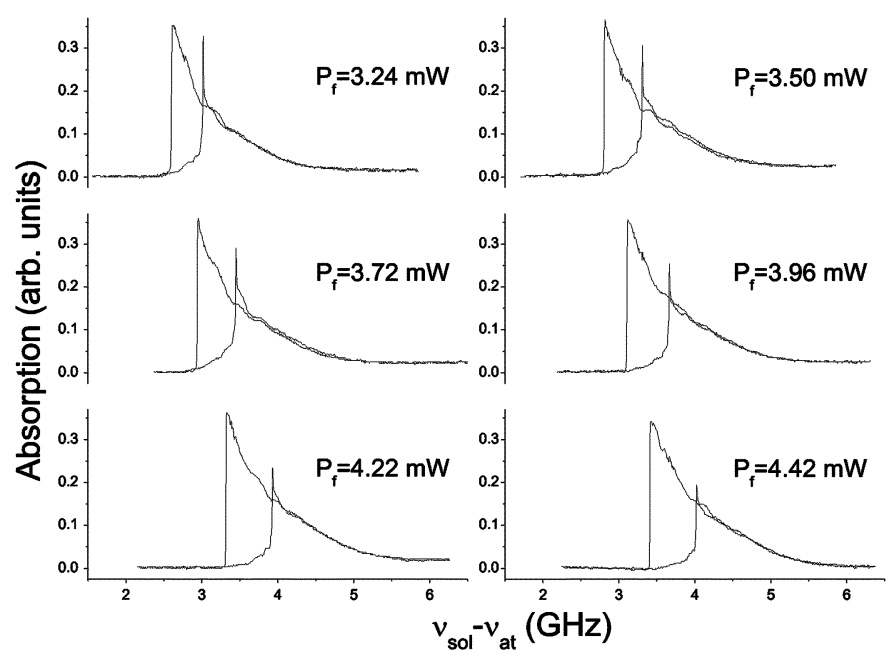

Fig. 8. Absorption spectra for high feedback power $\mathcal{P}_{f}$. $\mathcal{P}_{f}$ values are indicated at the top of each spectrum. $\beta=1.23 \mathrm{GHz} / \mathrm{mW}$.

of Fig. 9(b), the second peak of the spectrum is not so narrow, but its amplitude is the same as the larger one. In Fig. 9(a), we show a spectrum qualitatively similar to the calculated in Fig. 3(b), which also presents the expected amplitude. All these results are in good agreement with the spectra calculated with the above model.

In Fig. 10 we plot, as a function of the orthogonal feedback intensity, the width of the frequency range of bistability. The increase of the hysteresis region is linear, as expected from the model presented here, because the frequency switch depends only on the position where the filter lineshape changes its derivative, this position being linearly shifted with the feedback power [see (20)]. The linear interpolation was made within less than $5 \%$ of the measured data of both axis, notwithstanding the two series are done with two different coupling coefficients.

In the experimental data analysis we eliminated an intrinsic scanning hysteresis, due to the thermal effects included in the model presented above. In order to quantify their contribution we performed measurements of up-down scanning, without

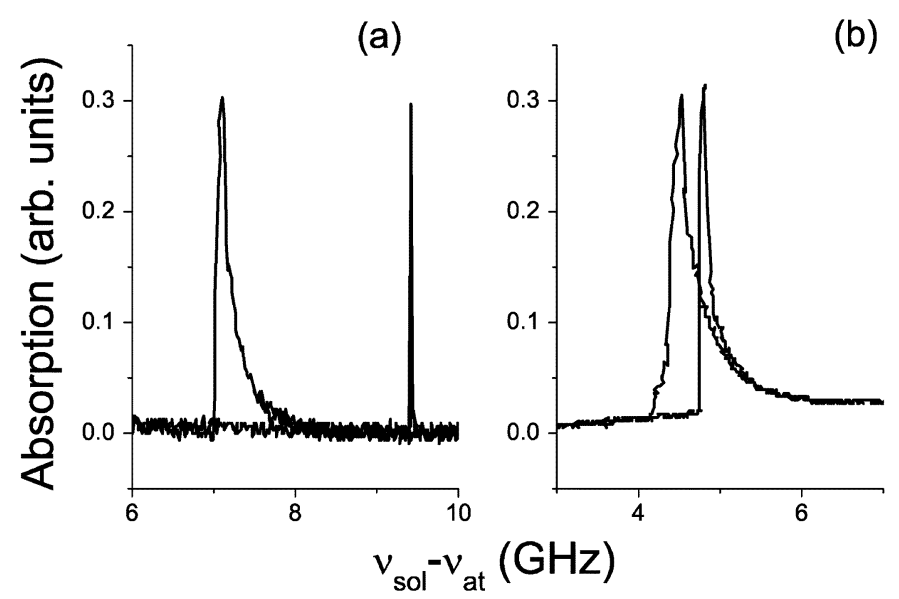

Fig. 9. Absorption spectra obtained with slow scanning of the laser current. The narrow peak has the same amplitude than the large one. (a) $\mathcal{P}_{f}=3.2 \mathrm{~mW}$ and $\beta=2.2 \mathrm{GHz} / \mathrm{mW}$; (b) $\mathcal{P}_{f}=3.5 \mathrm{~mW}$ and $\beta=1.3 \mathrm{GHz} / \mathrm{mW}$.

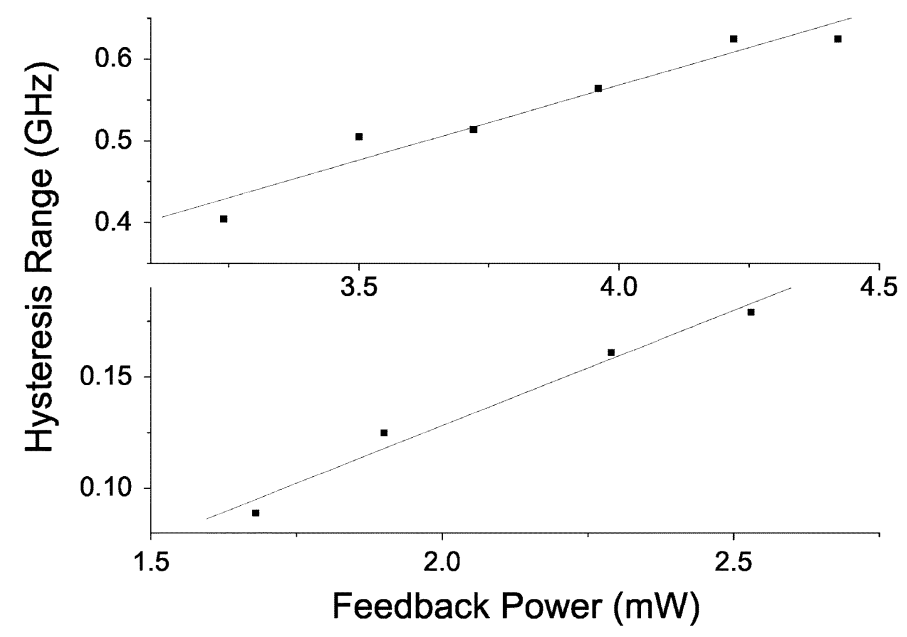

Fig. 10. Range of frequency bistability as a function of the feedback power. The upper curve corresponds to the absorption spectra of Fig. 8, the bottom curve corresponds to the spectra of Fig. 7. The different slopes are due to the different values of $\beta$.

feedback, using different rates for the current variation. The spectra obtained (Fig. 11) show a clear increase of the two peak separation when the scanning becomes faster. Comparing them with the theoretical curves of Fig. 5 we get a thermal response time in the time scale of milliseconds. This behavior confirms the importance of thermal effects in the laser frequency dynamics, supporting the model developed here.

It is interesting to establish a comparison between the dynamical response of semiconductor lasers under filtered parallel and orthogonal feedback. In the case of parallel polarization, due to coherent coupling of the feedback field with the one oscillating inside the semiconductor cavity, feedback levels as low as $-55 \mathrm{~dB}$ are enough to drastically change the laser emission characteristics [21]. This high sensitivity may be used to favor a more stable emission of the diode laser, with a narrower linewidth, but a strict control of the field phase is needed. In the case of filtered coherent feedback [22] practical applications demand a mechanism to keep constant the distance between the laser and the external filter. The feedback limit, first reported in 


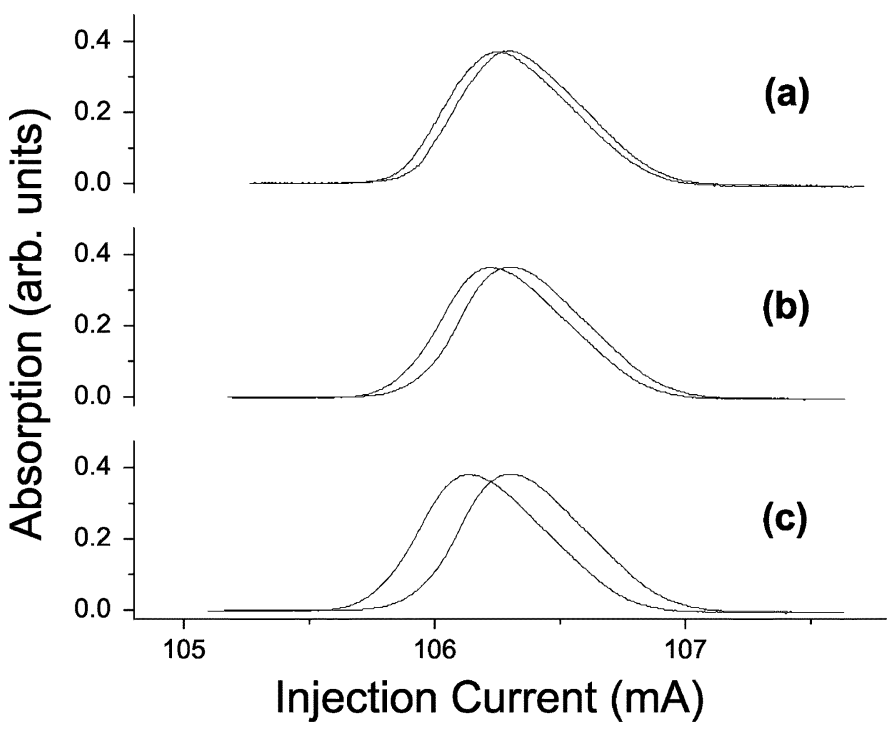

Fig. 11. Transmission spectra of the analysis cell without feedback. Scanning frequencies are: (a) $1 \mathrm{~Hz}$, (b) $10 \mathrm{~Hz}$, and (c) $30 \mathrm{~Hz}$.

[23], of about $-30 \mathrm{~dB}$ to have stable behavior in the laser emission under parallel filtered feedback was recently confirmed by Yousefi and collaborators [24] that observe that above $-26 \mathrm{~dB}$ the laser becomes multimode and may present frequency jumps. On the contrary, orthogonal feedback allows frequency change without the need of a precise control of the phase of the returned field, and the supported intensity levels are of the same order of magnitude as the total output power of the laser (for example, in this work the feedback level changes from about -16 to $-10 \mathrm{~dB}$ ).

\section{SUMMARY AND CONCLUSION}

We formulated a model to describe the dynamics of a single-longitudinal-mode diode laser subject to orthogonal time-delayed optical feedback. The model includes thermal and gain-saturation effects. Thermal effects are taken into account via a rate equation for the lattice temperature, describing Joule heating, carrier heating, and heat dissipation towards the environment. The temperature dynamics is coupled to the TE field dynamics via the linear shift of the cavity resonance (4). Gain saturation effects are taken into account via the usual self- and cross- saturation coefficients (13). The orthogonal feedback, that does not interfere coherently with the lasing TE field, but that depletes the shared carrier reservoir, was taken into account through a time delayed term in the carrier rate equation. The strength of the feedback was modulated by a frequency-dependent Gaussian filter.

Within the framework of this model we proposed an explanation for the origin of the linear relation between feedback-induced frequency shift and the feedback strength, observed experimentally in [18]. The model is tested by reproducing new experimental conditions, particularly exploring the coupling strength of the orthogonal feedback field.

The numerical simulations show that orthogonal feedback leads to a region of frequency bistability with almost constant output power, and an increase of the feedback strength increases the size of the bistability domain, also in good agreement with the observations.

For large enough feedback the frequency-dependent filter introduces an oscillatory instability, which is not present if the feedback strength is independent of the frequency of the feedback beam. The numerical simulations show that the oscillatory instability disappears and the laser output is stable if the filter responds to the time-averaged frequency instead of the instantaneous frequency of the feedback field.

By using an atomic filter with a larger width, it would be possible to increase the frequency bistability range, without having as a drawback the increase of the output power modulation and the laser instabilities. This opens the possibility of applications to optical switching [26] and to coherent communications requiring precise carrier frequencies [27], [28], which may be obtained by frequency-locking the laser on to stable references such as atomic or molecular transitions.

It is also predicted numerically and verified experimentally that the presence of thermal effects introduces hysteresis in the absorption spectrum even in the absence of orthogonal feedback, the hysteresis region depending on the velocity of the scan of the laser frequency across the filter frequency.

\section{REFERENCES}

[1] D. Lenstra, B. H. Verbeek, and A. J. Denboef, "Coherence collapse in single-mode semiconductor-lasers due to optical feedback," IEEE $J$. Quantum Electron., vol. 21, no. 6, pp. 674-679, Jun. 1985.

[2] J. Mork, B. Tromborg, and J. Mark, "Chaos in semiconductor-lasers with optical feedback-theory and experiment," IEEE J. Quantum Electron., vol. 28, no. 1, pp. 93-108, Jan. 1992.

[3] J. Ohtsubo, , E. Wolf, Ed., Progress in Optics. Amsterdam, The Netherlands: North-Holland, 2002, vol. 44, ch. 1.

[4] T.-C. Yen, J.-W. Chang, J.-M. Lin, and R.-J. Chen, "High-frequency optical signal generation in a semiconductor laser by incoherent optical feedback," Opt. Commun., vol. 150, pp. 158-162, 1998.

[5] R. Ju and P. S. Spencer, "Dynamic regimes in semiconductor lasers subject to incoherent optical feedback," J. Lightw. Tech., vol. 23, no. 8, pp. 2513-2523, Aug. 2005

[6] D.-L. Cheng, T.-C. Yen, J.-W. Chang, and J.-K. Tsai, "Generation of high-speed single-wavelength optical pulses in semiconductor lasers with orthogonal-polarization optical feedback," Opt. Commun., vol. 222, pp. 363-369, 2003

[7] D.-L. Cheng, T.-C. Yen, E.-C. Liu, and K.-L. Chuang, "Suppressing mode hopping in semiconductor lasers by orthogonal-polarization optical feedback," IEEE Photon. Technol. Lett., vol. 16, no. 6, pp. 1435-1437, Jun. 2004.

[8] A. Gavrielides, T. Erneux, D. W. Sukow, G. Burner, T. McLachlan, J. Miller, and J. Amonette, "Square-wave self-modulation in diode lasers with polarization-rotated optical feedback," Opt. Lett., vol. 31, pp. 2006-2009, 2007.

[9] W. H. Loh, A. T. Schremer, and C. L. Tang, "Polarization self-modulation at multigigahertz frequencies in an external-cavity semiconductor-laser," IEEE Photon. Technol. Lett., vol. 2, pp. 467-469, 1990.

[10] W. H. Loh, Y. Ozeki, and C. L. Tang, "High-frequency polarization self-modulation and chaotic phenomena in external cavity semiconductor-lasers," Appl. Phys. Lett., vol. 56, pp. 2613-2615, 1990.

[11] M. Sciamanna, F. Rogister, O. Deparis, P. Megret, M. Blondel, and T. Erneux, "Bifurcation to polarization self-modulation in vertical-cavity surface-emitting lasers," Opt. Lett., vol. 27, pp. 261-263, 2002.

[12] Y. H. Hong, M. W. Lee, P. S. Spencer, and K. A. Shore, "Synchronization of chaos in unidirectionally coupled vertical-cavity surface-emitting semiconductor lasers," Opt. Lett., vol. 29, pp. 1215-1217, 2004.

[13] R. Ju, P. S. Spencer, and K. A. Shore, "Polarization-preserved and polarization-rotated synchronization of chaotic vertical-cavity surface-emitting lasers," IEEE J. Quantum. Electron., vol. 41, no. 12, pp. 1461-1467, Dec. 2005. 
[14] D. W. Sukow, K. I. Blackburn, A. R. Spain, K. J. Babcock, J. V. Bennett, and A. Gavrielides, "Experimental synchronization of chaos in diode lasers with polarization-rotated feedback and injection," Opt. Lett., vol. 29, pp. 2393-2395, 2004.

[15] D. W. Sukow, A. Gavrielides, T. Erneux, M. J. Baracco, Z. A. Parmenter, and K. L. Blackburn, "Two-field description of chaos synchronization in diode lasers with incoherent optical feedback and injection," Phys. Rev. A, vol. 74, p. 043818, 2005.

[16] N. Shibasaki, A. Uchida, S. Yoshimori, and P. Davis, "Characteristics of chaos synchronization in semiconductor lasers subject to polarization-rotated optical feedback," IEEE J. Quantum Electron., vol. 42, no. 3, pp. 342-350, Mar. 2007.

[17] A. F. A. da Rocha, P. C. S. Segundo, M. Chevrollier, and M. Oria, "Diode laser coupled to an atomic line by incoherent optical negative feedback," Appl. Phys. Lett., vol. 84, pp. 179-181, 2004.

[18] B. Farias, T. P. de Silans, M. Chevrollier, and M. Oria, "Frequency bistability of a semiconductor laser under a frequency-dependent feedback," Phys. Rev. Lett., vol. 94, pp. 173902-, 2005.

[19] A. J. Scroggie, J. M. McSloy, and W. J. Firth, "Self-propelled cavity solitons in semiconductor microcavities," Phys. Rev. E, vol. 66, p. 036607, 2002

[20] L. Spinelli, G. Tissoni, L. A. Lugiato, and M. Brambilla, "Thermal effects and transverse structures in semiconductor microcavities with population inversion," Phys. Rev. A, vol. 66, p. 023817, 2002.

[21] R. W. Tkach and A. R. Chraplyvy, "Regimes of feedback effects in 1.5m DFB lasers," J. Lightw. Technol., vol. LT-4, no. 11, p. 1655, Nov. 1986.

[22] H. Erzgrber, B. Krauskopf, D. Lenstra, A. P. A. Fischer, and G. Vemuri, "Frequency versus relaxation oscillations in a semiconductor laser with coherent filtered optical feedback," Phys. Rev. E, vol. 73, p. 055201(R), 2007.

[23] P. Laurent, A. Clairon, and C. Breant, "Frequency noise analysis of optically self-locked diode lasers," IEEE J. Quantum Electron., vol. 25, no. 6, pp. 1131-1142, Jun. 1989.

[24] A. P. A. Fischer, O. K. Andersen, M. Yousefi, S. Stolte, and D. Lenstra, "Experimental and theoretical study of filtered optical feedback in a semiconductor laser," IEEE J. Quantum Electron., vol. 36, no. 3, pp. 375-384, Mar. 2000.

[25] M. Oria, B. Farias, T. Sorrentino, and M. Chevrollier, Submitted to publication, 2006.

[26] F. Prati, M. Travagnin, and L. A. Lugiato, "Logic gates and optical switching with vertical-cavity surface-emitting lasers," Phys. Rev. A, vol. 55, pp. 690-700, 1997.
[27] M. Tetu, B. Villeneuve, N. Cyr, P. Tremblay, S. Theriault, and M. Breton, "Multiwavelength sources using laser-diodes frequency-locked to atomic resonances," J. Lightw. Tech., vol. 7, no. 10 , pp. $1540-1548$, Oct. 1989.

[28] D. Psaltis, “Coherent optical information systems," Science, vol. 298, pp. 1359-1363, 2002.

C. Masoller was born in Montevideo, Uruguay, in 1963. She received the M.Sc. degree in physics from the Universidad de la Republica, Montevideo, Uruguay, in 1991 and the Ph.D. degree in physics from Bryn Mawr College, Bryn Mawr, PA, in 1999.

Since 2004, she has been a Ramon and Cajal Researcher at the Universitat Politecnica de Cataluña, Cataluña, Spain. Her research interests include theoretical modelling of nonlinear dynamics of lasers, synchronization and stochastic phenomena, and time-delayed systems.

T. Sorrentino was born in 1983, in Joao Pessoa, Brazil. He is an undergraduate student at the Universidade Federal da Paraíba, where he works with semiconductor laser instrumentation and frequency dynamics under orthogonal optical feedback, in the group of Prof. M. Oria.

M. Chevrollier received the Ph.D. degree in atomic physics from the University of Paris XIII, France, in 1992.

In 1999, she became a Professor at the Universidade Federal da Paraiba, Joao Pessoa, Brazil. Her research interests include interactions between atoms and surfaces, laser spectroscopy, and more recently, frequency dynamics of semiconductor lasers with orthogonal optical feedback.

M. Oria was born in 1958, in Paulo Afonso, Brazil. He received the Ph.D. degree in atomic physics from the University of Paris XIII, France, in 1990.

He became a Professor at the Universidade Federal da Paraiba, Joao Pessoa, Brazil, in 1994. His research interests include interactions between atoms and surfaces, and semiconductor lasers dynamics. 